\title{
BASES OF TYPE $P$ AND REFLEXIVITY OF BANACH SPACES
}

\author{
J. R. HOLUB
}

Abstract. The purpose of this paper is to give a number of characterizations of reflexive Banach spaces having Schauder bases.

We show that a Banach space $X$ with a basis such that $c_{0}$ is contained in either $X$ or in $X^{*}$ has a basis of type $P$. It follows that a Banach space with an unconditional basis is reflexive if and only if no basis is of type $P$. It is also shown that a Banach space with a basis is reflexive if and only if every basis converges weakly to zero.

1. Introduction. In [10] Singer has given the following characterization of reflexive Banach spaces with bases:

Theorem A. A Banach space $X$ with a basis is reflexive if and only if no basic sequence in $X$ is of type $P$ (equivalently; type $P^{*}$, type $l_{+}$) (see $\$ 2$ for definitions).

An immediate consequence of this theorem is

Theorem B. A Banach space $X$ with a basis is reflexive if and only if every basic sequence is of type wc $w_{0}$ (see \$2).

In the same paper Singer has asked whether these characterizations of reflexivity also hold true if the expression "basic sequence" is replaced by "basis for $X$ " [10, p. 368]. Answering a question raised by Pełczyński, Foiaş and Singer have shown that $C[0,1]$ has a basis of type $P$ and have asked whether $L^{1}[0,1]$ has such a basis [3].

In $\$ 3$ we use the "extension lemma" of Zippin [11] to give a partial solution to the general problem of Singer by showing that if $X$ is a nonreflexive Banach space with a basis such that $c_{0}$ is contained in either $X$ or $X^{*}$, then $X$ has a basis of type $P$ (equivalently, a basis of type $P^{*}, l_{+}$). In the case where $X$ may be embedded in a Banach space with an unconditional basis it follows that $X$ is nonreflexive if and only if $X$ has a basis of type $P$ (equivalently, type $P^{*}, l_{+}$).

In $\$ 4$ we show that in Theorem $B$ the expression "basic sequence" may indeed be replaced by "basis for $X$ ", and that if $X$ has an unconditional basis a stronger result may be obtained. 1969.

Presented to the Society, March 27, 1970; received by the editors November 17,

AMS Subject Classifications. Primary 4610, 4620; Secondary 4601.

Key Words and Phrases. Schauder basis, basic sequence, basis of type $P$, reflexive Banach space, unconditional basis, basis of type $w c_{0}$, weak convergence of bases. 
2. Definitions and preliminary results. A Schauder basis $\left(x_{i}\right)$ for a Banach space $X$ having coefficient functionals $\left(f_{i}\right)$ in $X^{*}$ and such that $0<\inf _{i}\left\|x_{i}\right\| \leqq \sup _{i}\left\|x_{i}\right\|<+\infty$ is said to be

(a) of type $P[10]$, if $\sup _{n}\left\|\sum_{i=1}^{n} x_{i}\right\|<+\infty$.

(b) of type $P^{*}[10]$, if $\sup _{n}\left\|\mid \sum_{i=1}^{n} f_{i}\right\|<+\infty$.

(c) of type $l_{+}[10]$, if there exists a constant $C>0$ such that for all finite sequences $a_{1}, a_{2}, \cdots, a_{n}$ for which $a_{i} \geqq 0$ for all $i$,

$$
\left\|\sum_{i=1}^{n} a_{i} x_{i}\right\| \geqq C \sum_{i=1}^{n} a_{i}
$$

(d) of type $w c_{0}[3]$, if the sequence $\left\{x_{i}\right\}$ converges weakly to zero in $X$.

(e) of type $\operatorname{swc}_{0}[3]$, if there is a subsequence $\left\{x_{n_{i}}\right\}$ of $\left\{x_{i}\right\}$ which converges weakly to zero in $X$.

(f) shrinking [6], if $\left(f_{i}\right)$ is a basis for $X^{*}$.

It is well known that in a Banach space $X$ the existence of a basis of type $P, P^{*}$, or $l_{+}$is equivalent to the existence in $X$ of each of the remaining two types [10]. Also, the basis $\left(x_{i}\right)$ is of type $P$ (of type $P^{*}$ ) if and only if the basic sequence $\left(f_{i}\right)$ in $X^{*}$ is of type $P^{*}$ (type $P$ ) [10].

If $\left\{y_{i}\right\}$ is a sequence in a Banach space $Y$ we denote by $\left[y_{i}\right]$ the closed linear span of the set $\left\{y_{i}\right\}$. If $X$ is isomorphic to a closed subspace of $Y$ we write $X \subset Y$, while if $X$ and $Y$ are isomorphic we write $X=Y$.

The only bases $\left(x_{i}\right)$ we will consider in this paper are those for which $0<\inf _{i}\left\|x_{i}\right\| \leqq \sup _{i}\left\|x_{i}\right\|<+\infty$.

\section{The main result.}

Theorem 3.1. Let $X$ be a Banach space with a basis such that $c_{0} \subset X$. Then $X$ has a basis of type $P$ (equivalently, of type $P^{*}, l_{+}$).

Proof. Suppose $\left(x_{i}\right)$ is a basis for $X$. Since $c_{0} \subset X$ and the unit vector basis for $c_{0}$ converges weakly to zero, it follows from a theorem of Bessaga and Pełczyński [1] that there is a block basic sequence

$$
\left(\sum_{i=p_{k}+1}^{p_{k+1}} a_{i} x_{i}\right)_{k=0}^{\infty}
$$

with respect to $\left(x_{i}\right)$ which is similar to the unit vector basis of $c_{0}$. By a lemma of Zippin [11] there then exists a basis $\left(w_{j}, g_{j}\right)$ for $X$ such that the subsequence $\left(w_{p_{k}}\right)$ of $\left(w_{j}\right)$ is similar to the unit vector basis of $c_{0}$. Clearly we may assume $\left\|w_{j}\right\|=1$ for all $j$.

Now if $\left\{w_{j}\right\} \backslash\left\{w_{p_{k}}\right\}$ is a finite set then the basis $\left(w_{j}\right)$ is of type $P$. Therefore we may assume there are infinitely many elements of the set $\left\{w_{j}\right\}$ which are not in $\left\{w_{p_{k}}\right\}$. We will define a rearrangement of 
the basis $\left\{w_{j}\right\}$ which will also be a basis for $X$ and then "perturb" it to get the desired basis.

Suppose $w_{1} \notin\left(w_{p_{k}}\right)$ (if $w_{1}=w_{p_{1}}$ simply interchange the following definitions of $y_{2 k+1}$ and $\left.y_{2 k}\right)$. Then let $y_{1}=w_{1}, y_{2}=w_{p_{1}}$ and for $k \geqq 2$,

$y_{2 k-1}=w_{j}$, where $j$ is the smallest integer which has not appeared as an index $m$ on any $w_{m}$ occurring in the definition of $y_{1}, y_{2}, \cdots, y_{2 k-2}$. $y_{2 k}=w_{p_{n}}$, where $p_{n}$ is the smallest integer in the set $\left\{p_{k}\right\}$ which has not appeared as an index $m$ on any $w_{m}$ occurring in the definition of $y_{1}, y_{2}, \cdots, y_{2 k-1}$.

Now $\left(y_{i}\right)_{i=1}^{\infty}$ is simply a rearrangement of the basis $\left(w_{j}\right)$ for $X$, so $\left[y_{i}\right]=X$ and the corresponding rearrangement of the coefficient functionals $\left(g_{j}\right)$ associated with $\left(w_{j}\right)$, call it $\left(h_{j}\right)$, is a sequence biorthogonal to $\left(y_{i}\right)$. Also, if $\sum_{j=1}^{\infty} a_{j} w_{j} \in X$ then $\left(a_{p_{k}}\right)$ converges to zero, implying $\sum_{k=1}^{\infty} a_{p_{k}} w_{p_{k}} \in X$. Thus there exists a natural projection $P: X \rightarrow\left[w_{p_{k}}\right]$ (which is continuous since $\left(w_{p_{k}}\right)$ is similar to $\left(e_{i}\right)$ in $\left.c_{0}\right)$.

Let $x \in X$. Then for any $N$ it follows from the definition of the set $\left(y_{i}\right)$ that there exist numbers $r, m$, and $n$ for which

Hence

$$
\sum_{i=1}^{N} h_{i}(x) y_{i}=\sum_{i=1}^{r} g_{i}(x) w_{i}+\sum_{i=m}^{n} g_{p_{i}}(x) w_{p_{i}} .
$$

$$
\begin{aligned}
\left\|\sum_{i=1}^{N} h_{i}(x) y_{i}\right\| & \leqq\left\|\sum_{i=1}^{r} g_{i}(x) w_{i}\right\|+\left\|\sum_{i=m}^{n} g_{p_{i}}(x) w_{p_{i}}\right\| \\
& \leqq K\|x\|+2 K\|P\|\|x\|,
\end{aligned}
$$

where the number $K \geqq 1$ is given by Grinblyum's $K$-condition on the basis $\left(w_{i}\right)$ [4]. Therefore we see that the partial sum operators of the biorthogonal system $\left(y_{i}, h_{i}\right)$ are pointwise bounded, and since $\left[y_{i}\right]=X$ it follows that $\left(y_{i}, h_{i}\right)$ is a basis for $X$ [7].

By a theorem of Pełczyński and Singer [8] the sequence $\left(z_{i}\right)$ defined for $k \geqq 1$ by

$$
z_{2 k-1}=y_{2 k-1}, \quad z_{2 k}=y_{2 k}-y_{2 k-1}
$$

is a basis for $X$. But since $\left(y_{2 k}\right)$ is similar to the unit vector basis of $c_{0}$ (a type $P$ basis) and $\left\|y_{i}\right\|=1$ for all $i$, the basis $\left(z_{i}\right)$ defined above is of type $P$ and the theorem is proved.

Proposition 3.2. Let $X$ be a Banach space with a basis $\left(x_{i}, f_{i}\right)$ such that $c_{0} \subset\left[f_{i}\right] \subset X^{*}$. Then $X$ has a basis of type $P$ (equivalently, $P^{*}$, $\left.l_{+}\right)$.

Proof. Suppose $c_{0} \subset\left[f_{i}\right]$. Then as in the proof of Theorem 3.1 there exists a block basic sequence with respect to the basis $\left(f_{i}\right)$ of $\left[f_{i}\right]$ which is similar to the unit vector basis of $c_{0}$. By Zippin's lemma [11] 
this block basic sequence may be extended to a basis $\left(q_{j}\right)$ for $\left[f_{i}\right]$. An inspection of Zippin's proof shows that the closed linear span of the coefficient functionals associated with the basis $\left(q_{j}\right)$ is isomorphic to the closed linear span of the coefficient functionals associated with the basis $\left(f_{i}\right)$ of $\left[f_{i}\right]$ and hence to $X$ since the sequence of coefficient functionals associated with $\left(f_{i}\right)$ is similar to the basis $\left(x_{i}\right)$ for $X$.

Now we proceed exactly as in the proof of Theorem 3.1 to obtain the basis $\left(y_{i}, h_{i}\right)$ for $\left[f_{i}\right]$ which is a rearrangement of the basis $\left(q_{j}\right)$, and hence for which $\left[h_{i}\right]=X$ according to our previous remarks. Again, as in Theorem 3.1, we perturb the basis $\left(y_{i}, h_{i}\right)$ using the theorem of Pełczyński and Singer to obtain the basis $\left(z_{i}, z_{i}^{*}\right)$ for $\left[f_{i}\right]$ which is of type $P$. It is clear from Pełczyński and Singer's proof [8] that $\left[z_{i}^{*}\right]=\left[h_{i}\right]=X$. Therefore since $\left(z_{i}^{*}\right)$ is of type $P^{*}$ it must be that $X$ has a basis of type $P^{*}$ and hence a basis of type $P$.

Our next theorem shows that in Proposition 3.2 we need only assume $c_{0} \subset X^{*}$ to obtain the same result.

Theorem 3.3. Let $X$ be a Banach space with a basis such that $c_{0} \subset X^{*}$. Then $X$ has a basis of type $P$ (equivalently, $\left.P^{*}, l_{+}\right)$.

Proof. Let $\left(x_{i}, f_{i}\right)$ be a basis for $X$. Since $c_{0} \subset X^{*}$ there exists a complemented subspace of $X$ isomorphic to $l^{1}$ [1]. Thus we may write $X=l^{1} \oplus X_{1}$ for some subspace $X_{1}$ of $X$ and since $l^{1} \oplus l^{1}=l^{1}$ we have $X=l^{1} \oplus X_{1}=l^{1} \oplus\left(l^{1} \oplus X_{1}\right)=l^{1} \oplus X$.

It is well known that if $\left(e_{i}\right)$ denotes the unit vector basis for $l^{1}$ then the sequence $\left(z_{i}\right)$ defined by

$$
z_{: k-1}=\left(e_{k}, 0\right), \quad z_{: k}=\left(0, x_{k}\right)
$$

is a basis for $?^{1} \oplus X$ whose sequence of coefficient functionals is the sequence $\left(z_{1}^{*}\right)$ defined by

$$
\stackrel{*}{z_{2: k-1}}=\left(e_{k}, 0\right), \quad \stackrel{*}{z_{2 k}}=\left(0, f_{k}\right), \quad k=1,2, \cdots,
$$

where here $\left(e_{j}\right)$ denotes the unit vector basis for $c_{0}$. Therefore $c_{0} \subset\left[z_{1}^{*}\right]$ and by Proposition 3.2 the space $l^{1} \oplus X$ has a basis of type $P$. Since we have siown that $l^{1} \oplus X=X$ it follows that $X$ has a basis of type $P$.

As we have mentioned previously, Foiaş and Singer have constructed a basis for $C[0,1]$ of type $P^{P}$ and have asked whether $L^{1}[0,1]$ has such a basis. This last question has recently been answered afnrmatively [5] using a construction process. However we see that both of these results are immediate consequences of Theorems 3.1 and 3.3 since $c_{0} \subset C[0,1]$ and $c_{0} \subset\left(L^{1}[0,1]\right)^{*}$.

Of course there are nonreflexive Banach spaces with bases for which $c_{0}$ can not be embedded in either the space or in its dual (e.g. the 
space of James [6]), so Theorems 3.1 and 3.3 do not completely characterize nonreflexive Banach spaces with bases. However, as the next theorem shows, we are able to characterize nonreflexive Banach spaces with bases which may be embedded in a Banach space with an unconditional basis.

Theorem 3.4. Let $X$ be a Banach space with a basis such that $X$ can be embedded in a Banach space with an unconditional basis. Then $X$ is reflexive if and only if no basis for $X$ is of type $P$ (equivalently, $P^{*}, l_{+}$).

Proof. If $X$ is reflexive then no basic sequence is of type $P[10]$ so certainly no basis is of type $P$.

Suppose $X$ is nonreflexive. Then if $c_{0} \subset X$ it follows from Theorem 3.1 that $X$ has a basis of type $P$. If $c_{0} \nsubseteq X$, then $l^{1} \subset X[2]$ and hence $X$ contains a subspace isomorphic to $l^{1}$ and complemented in $X$ [1]. Hence $c_{0} \subset X^{*}[1]$ and by Theorem $3.3 X$ has a basis of type $P$.

Pełczyński and Singer have shown that every infinite dimensional Banach space with a basis has a conditional basis [8]. As a corollary of Theorem 3.4 we obtain the following special case of this result.

Corollary 3.5. Every nonreflexive Banach space $X$ with a basis has a conditional basis.

Proof. Suppose every basis for $X$ is unconditional. Then by Theorem 3.4 there is an unconditional basis for $X$ of type $P$ and so $X=c_{0}[10]$. But it is well known that $c_{0}$ has a conditional basis, a contradiction to our original assumption.

4. Our purpose in this section is to characterize reflexivity of Banach spaces with bases in terms of weak convergence of the bases. Our first result is a consequence of a theorem of Zippin [11] while our second follows from Theorem 3.4.

Theorem 4.1. Let $X$ be a Banach space with a basis. Then $X$ is reflexive if and only if every basis for $X$ is of type wc.

Proof. If $X$ is reflexive then no basic sequence is of type $l_{+}$[10] and so every basis is of type $w c_{0}$.

Suppose every basis is of type $w c_{0}$ and let $\left(x_{i}, f_{i}\right)$ be any basis for $X$. If $\left(x_{i}\right)$ is not shrinking then there exists a bounded sequence $\left(y_{k}\right)$ in $X$ such that $f_{i}\left(y_{k}\right) \rightarrow{ }^{k} 0$ for all $i$ but $\left(y_{k}\right)$ does not converge weakly to zero [9]. Hence there is an $f \in X^{*}$ such that the sequence $\left\{f\left(y_{k}\right)\right\}$ does not converge to zero, implying there exists a subsequence $\left(y_{k_{j}}\right)$ of $\left(y_{k}\right)$ for which $\left|f\left(y_{k_{j}}\right)\right| \geqq \epsilon>0$ for all $j$, but for which $f_{i}\left(y_{k_{j}}\right) \stackrel{j}{\rightarrow} 0$ for each $i$.

By the theorem of Bessaga and Pełczyński [1] there is a subsequence $\left(y_{m}\right)$ of $\left(y_{k_{j}}\right)$ which is a basic sequence similar to a block 
basic sequence with respect to the basis $\left(x_{i}\right)$. Clearly, then, this block basic sequence is not weakly convergent to zero. However by Zippin's extension lemma [11] this block basic sequence may be extended to a basis for $X$ which, by the previous remark, is certainly not of type $w c_{0}$. But by assumption every basis for $X$ is of type $w c_{0}$.

This contradiction shows that the basis $\left(x_{i}\right)$ is shrinking, and since $\left(x_{i}\right)$ was arbitrary it follows from Zippin's theorem [11] that $X$ is reflexive.

In the case where $X$ has an unconditional basis we get the following characterization of reflexivity which is a stronger result than Theorem 4.1 .

THEOREM 4.2. Let $X$ be a Banach space with an unconditional basis. Then $X$ is reflexive if and only if every basis for $X$ is of type swc.

Proor. As in Theorem 4.1, if $X$ is reflexive then every basis is of type $s w c_{0}$.

Suppose every basis is of type $s w c_{0}$. Then no basis is of type $P^{*}$, and hence no basis is of type $P$. By Theorem $3.4, X$ is reflexive.

\section{REFERENCES}

1. C. Bessaga and A. Pełczyński, On bases and unconditional convergence of series in Banach spaces, Studia Math. 17 (1958), 151-164. MR 22 \#5872.

2. - A generalization of results of $R$. C. James concerning absolute bases in Banach spaces, Studia Math. 17 (1958), 165-174. MR 22 \#5874.

3. C. Foias and I. Singer, On bases in $C([0,1])$ and $L^{1}([0,1])$, Rev. Roumaine Math. Pures Appl. 10 (1965), 931-960. MR 34 \#6509.

4. M. M. Grinblyum, On the representation of a space of type $B$ in the form of a direct sum of subspaces, Dokl. Akad. Nauk SSSR 70 (1950), 749-752. (Russian) MR 11, 525.

5. J. R. Holub, Some problems concerning bases in Banach spaces, Proc. Amer. Math. Soc. 23 (1969), 521-525.

6. R. C. James, Bases and reflexivity of Banach spaces, Ann. of Math. (2) 52 (1950), 518-527. MR 12, 616.

7. S. Karlin, Bases in Banach spaces, Duke Math. J. 15 (1948), 971-985. MR 10, 548.

8. A. Pełczyński and I. Singer, On non-equivalent bases and conditional bases in Banach spaces, Studia Math. 25 (1964/65), 5-25. MR 31 \#3831.

9. J. R. Retherford, Shrinking bases in Banach spaces, Amer. Math. Monthly 73 (1966), 841-846. MR 34 \#1831.

10. I. Singer, Basic sequences and reflexivity of Banach spaces, Studia Math. 21 (1961/62), 351-369. MR 26 \#155.

11. M. Zippin, A remark on bases and reflexivity in Banach spaces, Israel J. Math. 6 (1968), 74-79. MR 38 \#4972.

Virginia Polytechnic Institute, Blacksburg, Virginia 24061 